\title{
Evolutionary conservation of gene linkage
}

\author{
from E. H. R. Ford
}

THE science of chromosome mapping in mammals has grown explosively in the past 10 years, largely because of the development of the techniques of chromosome banding and of somatic cell hybridisation. In man, over 110 gene loci have been assigned to specific autosomes, and 100 more to the $\mathrm{X}$ chromosome (McKusick \& Ruddle Science 196, 390; 1977). This is the highest number of gene assignments for any mammal, and the next highest number is in the mouse. In both man and mouse a number of gene linkage groups have been established by family and breeding studies, and it is now possible to show that some of these have been conserved in both (and other) species during the course of evolution.

The technique of somatic cell hybridisation depends on the fusion of cells from two different species, following which the chromosomes of one of the two species 'segregate' (are preferentially removed from the hybrid cell). Normally if human and rodent cells are fused, the human chromosomes are discarded, and this has been invaluable in constructing the human chromosome map (since the gene loci for enzymes can be located by studying which human chromosomes remain after fusion with rodent cells deficient in that enzyme). However J. D. Minna and his co-workers discovered that if cultured human cells are fused with differentiated rodent cells, the rodent cells segregate, and this discovery has been as valuable in building up the mouse chromosome map as the original one was for the human map. Somatic cell hybridisation is also valuable for showing which genes are 'syntenic', that is, located on the same chromosome, whether or not they are linked (Renwick A. Rev. Genet. 5, 81; 1971). It does not however tell whether genes are linked, and if so how closely; this information must still be obtained by sexual genetics.

Now Lalley, Minna and Francke (page 160 of this issue of Nature; see also Proc. natn. Acad. Sci. U.S.A. 75, $2382 ; 1978)$, inspired by the recent finding that five mouse genes are syntenic, and that these are also syntenic in chromosome 1 in man (four of them being assigned to the short arm), have sought other examples of genes which are syntenic in both mouse and man. Following their assignment of seven more gene loci to specific mouse chromosomes by somatic cell hybridisation, they have

E. H. R. Ford is a Lecturer in Anatomy in the University of Cambridge. found evidence for synteny of four pairs of gene loci on four different human and mouse autosomes. When these findings are combined with previous ones by this and other groups, it seems that there is now evidence for nine pairs or groups of genes which are syntenic on different autosomes in both man and mouse, as well as a cluster of genes which is syntenic on the $\mathrm{X}$ chromosome in both species.

It has been known for some time that some groups of very closely linked genes are conserved together (and are therefore of course syntenic) in several species-for example the major histocompatibility complex in man, rhesus monkey and mouse, the four $\gamma$-immunoglobulin heavy chain genes in man, mouse and rabbit, pancreatic and salivary amylase genes (man, mouse, Drosophila) and the $\beta$ and $\gamma$ polypeptide chains of haemoglobin (man and mouse). A plausible explanation of this is that the genes concerned are closely related to each other both in structure and function, and may indeed have arisen during evolution by tandem repeats (Ohno Nature 244, 259; 1973). There is every reason to suppose that such groups would be conserved, both because they are so very closely linked and because they are functionally interrelated. But the new findings of Lalley et al. suggest that quite distantly linked genes may be kept togther on the same chromosome; for example the genes for phosphoglucomutase-2 and phosphogluconate dehydrogenase are 23 map units apart in the mouse and not detectably linked in man.

The evolutionary significance of the preservation or disruption of syntenic groups of genes is, as Lalley et al. say, not yet understood. Obviously during mammalian evolution much chopping and changing of chromosome segments has occurred (from reciprocal translocations and inversions for example). In closely-related species such as man and chimpanzee these can be analysed and largely explained, but when species are as distantly related as man and mouse, it is generally difficult if not impossible to make homologies between the chromosome banding patterns or to reconstruct the chromosomal changes which have taken place in the course of evolution. There is however one notable exception to this, which is the mammalian $\mathrm{X}$ chromosome; this shows similar banding in some quite distantly related species (for example man and horse). Here Ohno's law, which suggests that if genes are found to be present or linked on the $\mathrm{X}$ chromosome of one mammalian species, they may similarly be expected also to be present or linked on the $\mathrm{X}$ chromosomes of any other species appears on present evidence to have considerable (if not total) validity.

It may be that, as Lalley et al. suggest, chromosomal rearrangements which tend to preserve ancestral synteny are preferentially favoured in evolution. One obvious example of this is the Robertsonian translocation (centric fusion) whereby two acrocentric chromesomes combine to form a single metacentric. This is widely documented as one of the commonest evolutionary changes both in primates and in other mammalian groups, and it is noteworthy that it does not involve any rearrangement of linkage groups.

The development of gene maps in different species, which is now progressing so vigorously, is clearly going to add profoundly to our understanding of the mammalian genome, and of the underlying mechanisms of its evolution, as well as to our knowledge of the functional relationships between different genes and clusters of genes.

\section{Large-scale characteristics of the Galaxy}

from Victor Clube

NEARLY two hundred astronomers gathered recently at the University of Maryland for the 84th Symposium of the International Astronomical Union, entitled 'The Large-Scale Characteristics of The Galaxy'. The aim of the gathering was to service and tune up The Working Model of our Galaxy. The machinery had evidently sprung a good many leaks, and though this was not allowed to be a cause for too much public concern, all is not well.

The Model is basically of 1920 vintage though it was called in for a major overhaul in the $1950 \mathrm{~s}$. It is now showing signs of obsolescence. The Model is at heart a spheroidal distribution of stars circulating interminably in epicyclic orbits. By the 1950 s, developments in the theory of stellar evolution and the advance of radio astronomy had also required a

Victor Clube is a member of staff at the Royal Observatory, Edinburgh. 